KYUNGPOOK Math. J. 49(2009), 435-450

\title{
General Formulas for Explicit Evaluations of Ramanujan's Cubic Continued Fraction
}

Megadahalli Sidda Naika Mahadeva NaIKA*

Department of Mathematics, Bangalore University, Central College Campus, Bangalore560 001, INDIA

e-mail : msmnaika@rediffmail.com

Mugur Chinna Swamy MaheshKumar

Department of Mathematics, Bangalore University, Central College Campus, Bangalore560 001, INDIA

e-mail : softmahe@rediffmail.com

KuRADY SUSHAN BAIRY

Department of Mathematics, Bangalore University, Central College Campus, Bangalore560 001, INDIA

e-mail : ksbairy@rediffmail.com

ABSTRACT. On page 366 of his lost notebook [15], Ramanujan recorded a cubic continued fraction and several theorems analogous to Rogers-Ramanujan's continued fractions. In this paper, we derive several general formulas for explicit evaluations of Ramanujan's cubic continued fraction, several reciprocity theorems, two formulas connecting $V(q)$ and $V\left(q^{3}\right)$ and also establish some explicit evaluations using the values of remarkable product of theta-function.

\section{Introduction}

In Chapter 16, of his second notebook, Ramanujan defines his theta-function as

$$
\begin{aligned}
f(a, b) & =\sum_{n=-\infty}^{\infty} a^{\frac{n(n+1)}{2}} b^{\frac{n(n-1)}{2}},|a b|<1, \\
& =(-a ; a b)_{\infty}(-b ; a b)_{\infty}(a b ; a b)_{\infty},
\end{aligned}
$$

where

$$
(a ; q)_{\infty}=\prod_{n=1}^{\infty}\left(1-a q^{n-1}\right) .
$$

* Corresponding author.

Received February 16, 2008; accepted July 8, 2008.

2000 Mathematics Subject Classification: Primary 33D10, 11A55, 11F27.

Key words and phrases: cubic continued fraction, modular equation, theta-function. 
Following Ramanujan for $|q|<1$, we define

$$
\begin{gathered}
\varphi(q):=f(q, q)=\sum_{n=-\infty}^{\infty} q^{n^{2}}=\left(-q ; q^{2}\right)_{\infty}^{2}\left(q^{2} ; q^{2}\right)_{\infty}, \\
\psi(q):=f\left(q, q^{3}\right)=\sum_{n=0}^{\infty} q^{\frac{n(n+1)}{2}}=\frac{\left(q^{2} ; q^{2}\right)_{\infty}}{\left(q ; q^{2}\right)_{\infty}}, \\
f(-q):=f\left(-q,-q^{2}\right)=\sum_{n=-\infty}^{\infty}(-1)^{n} q^{\frac{n(3 n-1)}{2}}=(q ; q)_{\infty}
\end{gathered}
$$

and

$$
\chi(q):=\left(-q ; q^{2}\right)_{\infty} .
$$

On page 366 of his lost notebook [15], Ramanujan recorded the cubic continued fraction

$$
V:=V(q):=\frac{q^{\frac{1}{3}}}{1}+\frac{q+q^{2}}{1}+\frac{q^{2}+q^{4}}{1}+\cdots, \quad|q|<1,
$$

and claimed that there are many results of $V(q)$ which are analogous to the RogersRamanujan continued fraction. H. H. Chan [7] established the relation between $V(q)$ and the other three continued fractions $V(-q), V\left(q^{2}\right)$ and $V\left(q^{3}\right)$. N. D. Baruah [3] established the relation between $V(q)$ and the two continued fractions $V\left(q^{5}\right)$ and $V\left(q^{7}\right)$.

In [4], Baruah and Nipen Saikia have also established some general theorems to find explicit evaluations of cubic continued fraction. In [1], C. Adiga, T. Kim, M. S. Mahadeva Naika and H. S. Madhusudan derived a modular equation relating $V(q)$ with $V\left(q^{3}\right)$, a transformation formula and several explicit evaluations of $V(q)$. For more discussions of (1.5), see [2] and [9].

On page 338 in his first notebook [14], Ramanujan defined

$$
a_{M, N}=\frac{N e^{-\frac{(N-1) \pi}{4} \sqrt{\frac{M}{N}}} \psi^{2}\left(e^{-\pi \sqrt{M N}}\right) \varphi^{2}\left(-e^{-2 \pi \sqrt{M N}}\right)}{\psi^{2}\left(e^{-\pi \sqrt{\frac{M}{N}}}\right) \varphi^{2}\left(-e^{-2 \pi \sqrt{\frac{M}{N}}}\right)} .
$$

He then, on pages 338 and 339, offered a list of eighteen particular values. All these eighteen values have been established by Berndt, Chan and Zhang [6]. In [10] and [11], Mahadeva Naika et al. derived general theorems to establish explicit new evaluations of Ramanujan's remarkable product of theta-function. In [12], Mahadeva Naika and M. C. Maheshkumar have also obtained other explicit evaluations of Ramanujan's remarkable product of theta-function. 
In [13], Mahadeva Naika, Maheshkumar and K. Sushan Bairy defined

$$
b_{M, N}=\frac{N e^{-\frac{(N-1) \pi}{4} \sqrt{\frac{M}{N}}} \psi^{2}\left(-e^{-\pi \sqrt{M N}}\right) \varphi^{2}\left(-e^{-2 \pi \sqrt{M N}}\right)}{\psi^{2}\left(-e^{-\pi \sqrt{\frac{M}{N}}}\right) \varphi^{2}\left(-e^{-2 \pi \sqrt{\frac{M}{N}}}\right)} .
$$

and established several general formulas for evaluating $b_{M, N}$.

The ordinary hypergeometric series ${ }_{2} F_{1}(a, b ; c ; x)$ is defined for $|x|<1$,

$$
{ }_{2} F_{1}(a, b ; c ; x)=\sum_{n=0}^{\infty} \frac{(a)_{n}(b)_{n} x^{n}}{(c)_{n} n !},
$$

where

$$
(a)_{0}=1 \text { and }(a)_{n}=a(a+1)(a+2) \cdots(a+n-1), \text { for } n \geq 1 .
$$

Let

$$
z(r):=z(r ; x):={ }_{2} F_{1}\left(\frac{1}{r}, \frac{r-1}{r} ; 1 ; x\right)
$$

and

$$
q_{r}:=q_{r}(x):=\exp \left(-\pi c s c\left(\frac{\pi}{r}\right) \frac{{ }_{2} F_{1}\left(\frac{1}{r}, \frac{r-1}{r} ; 1 ; 1-x\right)}{{ }_{2} F_{1}\left(\frac{1}{r}, \frac{r-1}{r} ; 1 ; x\right)}\right),
$$

where $r=2,3,4,6$ and $0<x<1$.

Let $N$ denote a fixed natural number, and assume that

$$
N \frac{{ }_{2} F_{1}\left(\frac{1}{r}, \frac{r-1}{r} ; 1 ; 1-\alpha\right)}{{ }_{2} F_{1}\left(\frac{1}{r}, \frac{r-1}{r} ; 1 ; \alpha\right)}=\frac{{ }_{2} F_{1}\left(\frac{1}{r}, \frac{r-1}{r} ; 1 ; 1-\beta\right)}{{ }_{2} F_{1}\left(\frac{1}{r}, \frac{r-1}{r} ; 1 ; \beta\right)},
$$

where $r=2,3,4$ or 6 . Then a modular equation of degree $N$ in the theory of elliptic functions of signature $r$ is a relation between $\alpha$ and $\beta$ induced by (1.9). We often say that $\beta$ is of degree $N$ over $\alpha$ and $m(r):=\frac{z(r: \alpha)}{z(r: \beta)}$ is called the multiplier. We also use the notations $z_{1}:=z_{1}(r)=z(r: \alpha)$ and $z_{N}:=z_{N}(r)=z(r: \beta)$ to indicate that $\beta$ has degree $N$ over $\alpha$. When the context is clear, we omit the argument $r$ in $q_{r}, z(r)$ and $m(r)$.

In Section 2, we collect some preliminary results which are useful in proving our main results. In Section 3, we establish our main results. In Section 4, we prove several reciprocity theorems for $V(q)$. In Section 5, we establish two formulas connecting $V(q)$ and $V\left(q^{3}\right)$. In Section 6, we also establish several explicit evaluations of $V(q)$.

\section{Preliminary results}

In this section, we collect some of the identities which are very useful to prove 
our main results.

Theorem 2.1. If $z$ is defined as in (1.8), then we have

$$
\begin{aligned}
& \varphi\left(e^{-y}\right)=\sqrt{z} \\
& \varphi\left(-e^{-2 y}\right)=\sqrt{z}(1-x)^{\frac{1}{8}} \\
& \psi\left(e^{-y}\right)=\sqrt{\frac{1}{2} z\left(x e^{y}\right)^{\frac{1}{8}}} \\
& \psi\left(-e^{-y}\right)=\sqrt{\frac{1}{2} z\left\{x(1-x) e^{y}\right\}^{\frac{1}{8}}} \\
& 1+\frac{1}{V}=\frac{\psi\left(q^{\frac{1}{3}}\right)}{q^{\frac{1}{3}} \psi\left(q^{3}\right)} \text { and } 1+\frac{1}{V^{3}}=\frac{\psi^{4}(q)}{q \psi^{4}\left(q^{3}\right)} \\
& 2 V=1-\frac{\varphi\left(-q^{\frac{1}{3}}\right)}{\varphi\left(-q^{3}\right)} \\
& 1-8 V^{3}=\frac{\varphi^{4}(-q)}{\varphi^{4}\left(-q^{3}\right)}, \text { where } V \text { is defined as in (1.5) }
\end{aligned}
$$

Proof. For a proof of (2.1) see [5, Entry 10(i), ch.17, p.122]; for a proof of (2.2) see [5, Entry 10(iii), ch.17, p.123]; for a proof of (2.3) see [5, Entry 11(i), ch.17, pp.123-124]; for a proof of (2.4) see [5, Entry 11(ii), ch.17, pp.123-124]; for a proof of (2.5) see [5, Entry 1(i), ch.20, pp.345-346]; for a proof of (2.6) see [5, Entry 1(ii), ch.20, pp.345-347]; for a proof of (2.7) see proof of Entry 1(iii) in [5, ch.20, p.347].

\section{General formulas for explicit evaluations of $V(q)$}

Lemma 3.1. If $\beta$ is of degree $N$ over $\alpha$, then

$$
\frac{N}{a_{M, N}}=m^{2}\left(\frac{\alpha(1-\alpha)}{\beta(1-\beta)}\right)^{\frac{1}{4}} .
$$

Proof. The identity (1.6) can be written as

$$
a_{M, N}=\frac{N q^{\frac{(N-1)}{4}} \psi^{2}\left(q^{N}\right) \varphi^{2}\left(-q^{2 N}\right)}{\psi^{2}(q) \varphi^{2}\left(-q^{2}\right)}, \quad q=e^{-\pi \sqrt{\frac{M}{N}}} .
$$

Using (2.2) and (2.3), we obtain (3.1).

Theorem 3.1. We have

$$
8 V^{3}\left(-e^{-\pi \sqrt{\frac{M}{3}}}\right)-\frac{1}{V^{3}\left(-e^{-\pi \sqrt{\frac{M}{3}}}\right)}+7=\frac{9}{a_{M, 3}^{2}} .
$$


Proof. Using (2.1) and (2.4) in (2.5) and (2.7), we find that

$$
-1-\frac{1}{V^{3}(-q)}=m^{2}\left(\frac{\alpha(1-\alpha)}{\beta(1-\beta)}\right)^{\frac{1}{2}}
$$

and

$$
1-8 V^{3}(-q)=\frac{z_{1}^{2}}{z_{3}^{2}}=m^{2}
$$

Using (3.4) and (3.5) in (3.1) with $N=3$, we obtain (3.3).

It is clear from the above Theorem 3.1 that to evaluate $V\left(-e^{-\pi \sqrt{\frac{M}{3}}}\right)$, one has to compute $a_{M, 3}$.

Corollary 3.1. We have

$$
V^{3}\left(-e^{-\frac{\pi}{\sqrt{3}}}\right)=-\frac{1}{4}
$$

Proof. Putting $M=1$ and $N=3$ in (3.2), we find that

$$
a_{1,3}=1 .
$$

Using (3.7) in (3.3), we obtain (3.6).

This was also evaluated by Adiga et al. [2] and Mahadeva Naika [9].

Corollary 3.2. We have

$$
V^{3}\left(-e^{-\pi}\right)=\frac{(5-3 \sqrt{3})}{4} .
$$

Proof. Putting $M=3$ and $N=3$ in (3.2), we find that

$$
a_{3,3}=\frac{1}{\sqrt{3}}
$$

Using (3.9) in (3.3), we obtain (3.8).

This was also evaluated by Baruah et al. [4] and Mahadeva Naika [9].

Theorem 3.2. We have

$$
2 V\left(-e^{-\pi \sqrt{M}}\right)-\frac{1}{V\left(-e^{-\pi \sqrt{M}}\right)}+1=\frac{3}{\sqrt{a_{M, 9}}} .
$$

Proof. Using (2.1) and (2.4) in (2.5) and (2.6), we find that

$$
1-2 V\left(-q^{3}\right)=\sqrt{\frac{z_{1}}{z_{9}}}=\sqrt{m} .
$$




$$
-1-\frac{1}{V\left(-q^{3}\right)}=\sqrt{m}\left(\frac{\alpha(1-\alpha)}{\beta(1-\beta)}\right)^{\frac{1}{8}} .
$$

and putting $N=9$ in (3.1), we find that

$$
\frac{9}{a_{M, 9}}=m^{2}\left(\frac{\alpha(1-\alpha)}{\beta(1-\beta)}\right)^{\frac{1}{4}}
$$

where $\beta$ is of degree 9 over $\alpha$. Using (3.11) and (3.12) in (3.13), we obtain (3.10).

It is clear from the above Theorem 3.2 that to evaluate $V\left(-e^{-\pi \sqrt{M}}\right)$, one has to compute $a_{M, 9}$.

Corollary 3.3. We have

$$
V\left(-e^{-\pi}\right)=\frac{1-\sqrt{3}}{2} .
$$

Proof. Putting $M=1$ and $N=9$ in (2.2), we find that

$$
a_{1,9}=1 .
$$

Using (3.15) in (3.10), we obtain (3.14).

This was also evaluated by Chan [7], Baruah et al. [4] and Mahadeva Naika [9].

Remark. In [9], Mahadeva Naika has also derived a different general formula to evaluate $V\left(-e^{-\pi \sqrt{n}}\right)$.

Theorem 3.3. We have

$$
\frac{1}{V^{3}\left(e^{-\pi \sqrt{\frac{M}{3}}}\right)}-8 V^{3}\left(e^{-\pi \sqrt{\frac{M}{3}}}\right)-7=\frac{9}{b_{M, 3}^{2}} .
$$

Proof. Using (2.1) and (2.4) in (2.5) and (2.7), we find that

$$
1+\frac{1}{V^{3}(q)}=m^{2}\left(\frac{\alpha}{\beta}\right)^{\frac{1}{2}}
$$

and

$$
1-8 V^{3}(q)=m^{2}\left(\frac{1-\alpha}{1-\beta}\right)
$$

where $\beta$ is of degree 3 over $\alpha$. Putting $N=3$ in (1.7) with $q=e^{-\pi \sqrt{\frac{M}{3}}}$ and then using (2.1) and (2.4), we find that

$$
\frac{9}{b_{M, 3}^{2}}=m^{4}\left(\frac{\alpha(1-\alpha)^{2}}{\beta(1-\beta)^{2}}\right)^{\frac{1}{2}} .
$$


Using (3.17) and (3.18) in (3.19), we obtain (3.16).

It is clear from the above Theorem 3.3 that to evaluate $V\left(e^{-\pi \sqrt{\frac{M}{3}}}\right)$, one has to compute $b_{M, 3}$.

Corollary 3.4. We have

$$
V^{3}\left(e^{-\sqrt{\frac{2}{3}} \pi}\right)=\frac{3-2 \sqrt{2}}{2 \sqrt{2}} .
$$

Proof. Putting $M=2$ and $N=3$ in (1.7), we find that

$$
b_{2,3}=1 \text {. }
$$

Using (3.21) in (3.16), we obtain (3.20).

Theorem 3.4. We have

$$
\frac{1}{V\left(e^{-\pi \sqrt{M}}\right)}-2 V\left(e^{-\pi \sqrt{M}}\right)-1=\frac{3}{\sqrt{b_{M, 9}}} .
$$

Proof. Using (2.1) and (2.4) in (2.5) and (2.6), we find that

$$
1-2 V\left(q^{3}\right)=\sqrt{m}\left(\frac{1-\alpha}{1-\beta}\right)^{\frac{1}{4}}
$$

and

$$
1+\frac{1}{V\left(q^{3}\right)}=\sqrt{m}\left(\frac{\alpha}{\beta}\right)^{\frac{1}{8}}
$$

and putting $N=9$ in (1.7) with $q=e^{-\pi \sqrt{\frac{M}{9}}}$ and then using (2.1) and (2.4), we find that

$$
\frac{9}{\sqrt{b_{M, 9}}}=m\left(\frac{\alpha(1-\alpha)^{2}}{\beta(1-\beta)^{2}}\right)^{\frac{1}{8}}
$$

where $\beta$ is of degree 9 over $\alpha$. Using (3.23), (3.24) in (3.25), we obtain (3.22).

It is clear from the above Theorem 3.4 that to evaluate $V\left(e^{-\pi \sqrt{M}}\right)$, one has to compute $b_{M, 9}$.

Corollary 3.5. We have

$$
V\left(e^{-\sqrt{2} \pi}\right)=\frac{\sqrt{3}-\sqrt{2}}{\sqrt{2}} .
$$

Proof. Putting $M=2$ and $N=9$ in (1.7), we find that

$$
b_{2,9}=1 .
$$


Using (3.27) in (3.22), we obtain (3.26).

This was also evaluated by Chan [7].

\section{Some reciprocity theorems for $V(q)$}

In this section, we establish several reciprocity theorems for $V(q)$.

Theorem 4.1. We have

$$
V^{3}\left(-e^{-\pi \sqrt{\frac{1}{3 M}}}\right)=\frac{1+V^{3}\left(-e^{-\pi \sqrt{\frac{M}{3}}}\right)}{-1+8 V^{3}\left(-e^{-\pi \sqrt{\frac{M}{3}}}\right)} .
$$

Proof. Replacing $M$ by $\frac{1}{M}$ in (3.3), we find that

$$
8 V^{3}\left(-e^{-\pi \sqrt{\frac{1}{3 M}}}\right)-\frac{1}{V^{3}\left(-e^{-\pi \sqrt{\frac{1}{3 M}}}\right)}+7=\frac{9}{a_{\frac{1}{M}, 3}^{2}} .
$$

Using (3.3) and (4.2), we deduce that

$$
\left(8 x-\frac{1}{x}+7\right)\left(8 y-\frac{1}{y}+7\right)=81, x=V^{3}\left(-e^{-\pi \sqrt{\frac{M}{3}}}\right), y=V^{3}\left(-e^{-\pi \sqrt{\frac{1}{3 M}}}\right) .
$$

On simplification of the above identity (4.3), we find that

$$
(8 x+8 x y+8 y-1)(-y+8 x y-x-1)=0 .
$$

The first factor does not vanish in the neighborhood of $q=e^{-\frac{\pi}{\sqrt{3}}}$. But the second factor vanishes in the neighborhood of $q=e^{-\frac{\pi}{\sqrt{3}}}$. So by the identity theorem the second factor vanishes identically. Hence, we complete the proof.

Corollary 4.1. We have

$$
V^{3}\left(-e^{-\frac{\pi}{3}}\right)=\frac{-(\sqrt{3}+1)}{4} .
$$

Proof. Putting $M=3$ in (4.1), we find that

$$
V^{3}\left(-e^{-\frac{\pi}{3}}\right)=\frac{1+V^{3}\left(-e^{-\pi}\right)}{-1+8 V^{3}\left(-e^{-\pi}\right)} .
$$

Using (3.14) in (4.6), we obtain (4.5).

Theorem 4.2. We have

$$
V\left(-e^{-\pi \sqrt{\frac{1}{M}}}\right)=\frac{1+V\left(-e^{-\pi \sqrt{M}}\right)}{-1+2 V\left(-e^{-\pi \sqrt{M}}\right)} .
$$


Proof. Replacing $M$ by $\frac{1}{M}$ in (3.10), we find that

$$
2 V\left(-e^{-\pi \sqrt{\frac{1}{M}}}\right)-\frac{1}{V\left(-e^{-\pi \sqrt{\frac{1}{M}}}\right)}+1=\frac{3}{\sqrt{a_{\frac{1}{M}, 9}}} .
$$

Using (3.10) and (4.8), we deduce that

$$
\left(2 x-\frac{1}{x}+1\right)\left(2 y-\frac{1}{y}+1\right)=9, x=V\left(-e^{-\pi \sqrt{M}}\right), y=V\left(-e^{-\pi \sqrt{\frac{1}{M}}}\right) .
$$

On simplification of the above identity (4.9), we find that

$$
(2 x+2 x y+2 y-1)(-y+2 x y-x-1)=0 .
$$

The first factor does not vanish in the neighborhood of $q=e^{-\pi}$. But the second factor vanishes in the neighborhood of $q=e^{-\pi}$. So by the identity theorem the second factor vanishes identically. Hence, we complete the proof.

Corollary 4.2. We have

$$
V\left(-e^{-3 \pi}\right)=\frac{-2+\sqrt[3]{2(\sqrt{3}+1)}}{2(1+\sqrt[3]{2(\sqrt{3}+1)})}
$$

Proof. Using (4.5) in (4.7) with $M=9$ and solving the resultant equation, we obtain (4.11).

Theorem 4.3. We have

$$
\frac{1}{8 V^{3}\left(e^{-\pi \sqrt{\frac{2}{3 M}}}\right)}=\frac{1+V^{3}\left(e^{-\pi \sqrt{\frac{2 M}{3}}}\right)}{1-8 V^{3}\left(e^{-\pi \sqrt{\frac{2 M}{3}}}\right)} .
$$

Proof. Replacing $M$ by $2 M$ in (3.16), we find that

$$
\frac{1}{V^{3}\left(e^{-\pi \sqrt{\frac{2 M}{3}}}\right)}-8 V^{3}\left(e^{-\pi \sqrt{\frac{2 M}{3}}}\right)-7=\frac{9}{b_{2 M, 3}^{2}} .
$$

Replacing $M$ by $\frac{1}{M}$ in (4.13), we find that

$$
\frac{1}{V^{3}\left(e^{-\pi \sqrt{\frac{2}{3 M}}}\right)}-8 V^{3}\left(e^{-\pi \sqrt{\frac{2}{3 M}}}\right)-7=\frac{9}{b_{\frac{2}{M}, 3}^{2}} .
$$

Using (4.13) and (4.14), we deduce that

$$
\left(\frac{1}{x}-8 x-7\right)\left(\frac{1}{y}-8 y-7\right)=81, x=V^{3}\left(e^{-\pi \sqrt{\frac{2 M}{3}}}\right), y=V^{3}\left(e^{-\pi \sqrt{\frac{2}{3 M}}}\right) .
$$


On simplification of the above identity (4.15), we find that

$$
(8 x+8 x y+8 y-1)(-y+8 x y-x-1)=0 .
$$

The second factor does not vanish in the neighborhood of $q=e^{-\pi \sqrt{\frac{2}{3}}}$. But the first factor vanishes in the neighborhood of $q=e^{-\pi \sqrt{\frac{2}{3}}}$. So by the identity theorem the first factor vanishes identically. Hence, we complete the proof.

Corollary 4.3. We have

$$
V^{3}\left(e^{-\frac{\pi \sqrt{2}}{3}}\right)=\frac{\sqrt{3}-\sqrt{2}}{2 \sqrt{2}} .
$$

Proof. Using (3.26) in (4.12) with $M=3$ and solving the resulting equation, we obtain the required result.

Theorem 4.4. We have

$$
\frac{1}{2 V\left(e^{-\pi \sqrt{\frac{2}{M}}}\right)}=\frac{1+V\left(e^{-\pi \sqrt{2 M}}\right)}{1-2 V\left(e^{-\pi \sqrt{2 M}}\right)} .
$$

Proof. Replacing $M$ by $2 M$ in (3.22), we find that

$$
\frac{1}{V\left(e^{-\pi \sqrt{2 M}}\right)}-2 V\left(e^{-\pi \sqrt{2 M}}\right)-1=\frac{3}{\sqrt{b_{2 M, 9}}} .
$$

Replacing $M$ by $\frac{1}{M}$ in (4.19),we find that

$$
\frac{1}{V\left(e^{-\pi \sqrt{\frac{2}{M}}}\right)}-2 V\left(e^{-\pi \sqrt{\frac{2}{M}}}\right)-1=\frac{3}{\sqrt{b_{\frac{2}{M}, 9}} .}
$$

Using (4.19) and (4.20), we deduce that

$$
\left(\frac{1}{x}-2 x-1\right)\left(\frac{1}{y}-2 y-1\right)=9, x=V\left(e^{-\pi \sqrt{2 M}}\right), y=V\left(e^{-\pi \sqrt{\frac{2}{M}}}\right) .
$$

On simplification of the above identity (4.21), we find that

$$
(2 x+2 x y+2 y-1)(-y+2 x y-x-1)=0 .
$$

The second factor does not vanish in the neighborhood of $q=e^{-\pi \sqrt{2}}$. But the first factor vanishes in the neighborhood of $q=e^{-\pi \sqrt{2}}$. So by the identity theorem the first factor vanishes identically. Hence, we complete the proof.

Corollary 4.4. We have

$$
V\left(e^{-\pi \sqrt{6}}\right)=\frac{1-\sqrt{2} \sqrt[3]{3-2 \sqrt{2}}}{\sqrt{2}(\sqrt{2}+\sqrt[3]{3-2 \sqrt{2}})}
$$


Proof. Using (3.20) in (4.18) with $M=3$ and solving the resulting equation, we obtain the result $(4.23)$.

\section{Formulas relating $V(q)$ and $V\left(q^{3}\right)$}

In this section, we prove two formulas relating $V(q)$ and $V\left(q^{3}\right)$.

Theorem 5.1. We have

$$
V\left(q^{3}\right)=\frac{-1+t}{1+2 t}
$$

where

$$
t=\sqrt[3]{\frac{1+V^{3}(q)}{1-8 V^{3}(q)}}
$$

Proof. Using (4.18) with replacing $M$ by $3 M$ in (4.12), we find that

$$
\frac{1+V\left(e^{-\pi \sqrt{6 M}}\right)}{1-2 V\left(e^{-\pi \sqrt{6 M}}\right)}=\sqrt[3]{\frac{1+V^{3}\left(e^{-\pi \sqrt{\frac{2 M}{3}}}\right)}{1-8 V^{3}\left(e^{-\pi \sqrt{\frac{2 M}{3}}}\right)}}
$$

On simplification of the above identity (5.2) with $q=e^{-\pi \sqrt{\frac{2 M}{3}}}$, we obtain the required result (5.1).

Replacing $q$ by $-q$ in Theorem 5.1 and observe that $t$ is equal to $-y$, then we obtain the following theorem.

Theorem 5.2. We have

$$
V\left(-q^{3}\right)=\frac{1+y}{-1+2 y}
$$

where

$$
y=\sqrt[3]{\frac{1+V^{3}(-q)}{-1+8 V^{3}(-q)}}
$$

\section{Some explicit evaluations of $V(q)$}

In this section, we establish following explicit evaluations of Ramanujan's cubic continued fractions.

Theorem 6.1. We have

$$
V^{3}\left(-e^{-\pi \sqrt{\frac{14}{3}}}\right)=\frac{-7 x^{2}+9-3 \sqrt{9 x^{4}-14 x^{2}+9}}{16 x^{2}}
$$


where

$$
\begin{aligned}
x= & \sqrt{\frac{4125 \sqrt{7}-10887-2376 \sqrt{21}+6300 \sqrt{3}}{2}} \\
& -\sqrt{\frac{4125 \sqrt{7}-10889-2376 \sqrt{21}+6300 \sqrt{3}}{2}} .
\end{aligned}
$$

$$
V^{3}\left(-e^{-\pi \sqrt{\frac{2}{3}}}\right)=\frac{9 \sqrt{6}-15 \sqrt{2}+12 \sqrt{3}-22}{4}
$$

$$
\begin{aligned}
& V\left(-e^{-\pi \sqrt{2}}\right) \\
& =\frac{1}{4}[\sqrt{97-51 \sqrt{3}-63 \sqrt{2}+39 \sqrt{6}}-\sqrt{105-51 \sqrt{3}-63 \sqrt{2}+39 \sqrt{6}}]
\end{aligned}
$$

and

$$
V\left(-e^{-\pi \sqrt{3}}\right)=\frac{1-\sqrt[3]{4}}{2+\sqrt[3]{4}}
$$

Proof of (6.1). From Ramanujan's first notebook [14, p.316], we have

$$
g_{42}=(2 \sqrt{2}+\sqrt{7})^{\frac{1}{6}}\left(\frac{\sqrt{3}+\sqrt{7}}{2}\right)^{\frac{1}{2}} .
$$

From the Theorem 4.1(i) in [8], we have

$$
2 \sqrt{2}\left[g_{n}^{3} g_{9 n}^{3}+\frac{1}{g_{n}^{3} g_{9 n}^{3}}\right]=\frac{g_{9 n}^{6}}{g_{n}^{6}}-\frac{g_{n}^{6}}{g_{9 n}^{6}} .
$$

Using (6.5) in (6.6) with $n=\frac{14}{3}$, we find that

$$
g_{\frac{14}{3}}=(2 \sqrt{2}-\sqrt{7})^{\frac{1}{6}}\left(\frac{\sqrt{3}+\sqrt{7}}{2}\right)^{\frac{1}{2}} .
$$

From the Theorem 4.3(i) in [8] with slight modification, we have

$$
G_{n} G_{9 n}=\left(2 g_{n} g_{9 n}\right)^{-\frac{1}{2}} \sqrt{g_{n}^{3} g_{9 n}^{3}+\sqrt{g_{n}^{6} g_{9 n}^{6}+2}} .
$$


Using (6.5) and (6.7) in (6.8) with $n=\frac{14}{3}$, we deduce that

$$
G_{\frac{14}{3}} G_{42}=(2+\sqrt{3})^{\frac{1}{2}}\left(\frac{3+\sqrt{7}}{2}\right)^{\frac{1}{2}}\left(\frac{\sqrt{7}-\sqrt{3}}{2}\right)^{\frac{1}{2}} .
$$

From the Theorem 4.2(i) in [8], we have

$$
2 \sqrt{2}\left[G_{n}^{3} G_{9 n}^{3}-\frac{1}{G_{n}^{3} G_{9 n}^{3}}\right]=\frac{G_{9 n}^{6}}{G_{n}^{6}}+\frac{G_{n}^{6}}{G_{9 n}^{6}} .
$$

Using (6.9) in (6.10) with $n=\frac{14}{3}$, we find that

$$
\begin{aligned}
\frac{G_{\frac{14}{3}}^{6}}{G_{42}^{6}}= & \sqrt{\frac{37125 \sqrt{7}+56700 \sqrt{3}-97999-21384 \sqrt{21}}{2}} \\
& -3 \sqrt{\frac{4125 \sqrt{7}+6300 \sqrt{3}-10889-2376 \sqrt{21}}{2}} .
\end{aligned}
$$

From Theorem 2.1 in [6], we have

$$
a_{M, 3}-\frac{1}{a_{M, 3}}=\frac{1}{3}\left[\frac{G_{\frac{M}{3}}^{6}}{G_{3 M}^{6}}-\frac{G_{3 M}^{6}}{G_{\frac{M}{3}}^{6}}\right] .
$$

Using (6.11) in (6.12) with $M=14$, we have

$$
\begin{aligned}
a_{14,3}= & \sqrt{\frac{4125 \sqrt{7}-10887-2376 \sqrt{21}+6300 \sqrt{3}}{2}} \\
& -\sqrt{\frac{4125 \sqrt{7}-10889-2376 \sqrt{21}+6300 \sqrt{3}}{2}} .
\end{aligned}
$$

Using (6.13) in (3.3), we obtain the required result.

As the proofs of the identities (6.2)-(6.4) being similar to the proof of the identity (6.1). So we omit the details.

Theorem 6.2. We have

$$
V^{3}\left(e^{-\pi \sqrt{\frac{5}{3}}}\right)=\frac{-209-93 \sqrt{5}+54 \sqrt{15}+120 \sqrt{3}}{2},
$$

$$
V^{3}\left(e^{-\pi \sqrt{8}}\right)=\frac{1}{8}(19-9 \sqrt{3}+9 \sqrt{6}-18 \sqrt{2})
$$




$$
\begin{aligned}
V^{3}\left(e^{-\pi \sqrt{10}}\right) & =\frac{1}{4 \sqrt[4]{5}}(158 \sqrt{2}+128 \sqrt{3})^{\frac{1}{4}} \\
& \times[\sqrt{6 \sqrt{10 \sqrt{3}+20 \sqrt{2}}+30 \sqrt[4]{3}}-\sqrt{2 \sqrt{2108 \sqrt{2}-1494 \sqrt{3}}+30 \sqrt[4]{3}}]
\end{aligned}
$$

and

$$
V^{3}\left(e^{-\pi \sqrt{\frac{7}{3}}}\right)=\frac{-\left(7 x^{2}+9\right)+3 \sqrt{9 x^{4}+14 x^{2}+9}}{16 x^{2}},
$$

where

$$
x=\sqrt{\frac{207+78 \sqrt{7}-3 \sqrt{9149+3458 \sqrt{7}}}{2}}-\sqrt{\frac{205+78 \sqrt{7}-3 \sqrt{9149+3458 \sqrt{7}}}{2}} .
$$

Proof of (6.14). From Ramanujan's first notebook [14, p.289], we have

$$
G_{15}=2^{\frac{1}{4}}\left(\frac{1+\sqrt{5}}{2}\right)^{\frac{1}{3}}
$$

Using (6.18) in (6.10) with $n=\frac{5}{3}$, we find that

$$
G_{\frac{5}{3}}=2^{\frac{1}{4}}\left(\frac{-1+\sqrt{5}}{2}\right)^{\frac{1}{3}}
$$

From Theorem 3.4(i) in [8], with replacing $q$ by $-q$ and using the fact that $g_{4 n}=2^{1 / 4} g_{n} G_{n}$, we deduce that

$$
g_{n}^{2} g_{9 n}^{2}=\frac{1}{2}\left[G_{n}^{2} G_{9 n}^{2}+\sqrt{G_{n}^{4} G_{9 n}^{4}-\frac{2}{G_{n}^{2} G_{9 n}^{2}}}\right] .
$$

Using (6.18) and (6.19) with $n=\frac{5}{3}$, we deduce that

$$
g_{15} g_{\frac{5}{3}}=\frac{\sqrt{3}+1}{2} .
$$

Using (6.21) in (6.6) with $n=\frac{5}{3}$, we find that

$$
\frac{g_{15}^{6}}{g_{\frac{5}{3}}^{6}}=\frac{1}{8}(\sqrt{5}-1)^{2}(9 \sqrt{6}+7 \sqrt{10})
$$


From Theorem 2.5 in [13], we have

$$
\frac{1}{b_{M, 3}}+b_{M, 3}=\frac{1}{3}\left(\frac{g_{3 M}^{6}}{g_{\frac{M}{3}}^{6}}+\frac{g_{\frac{M}{3}}^{6}}{g_{3 M}^{6}}\right) .
$$

Using (6.22) in (6.23) with $M=5$, we deduce that

$$
b_{5,3}=\frac{1}{4}(\sqrt{5}+\sqrt{3})(7 \sqrt{2}-3 \sqrt{10}) .
$$

Using (6.24) in (3.16), we obtain the required result.

As the proofs of the identities (6.15)-(6.17) being similar to the proof of the identity (6.14). So we omit the details.

Remark. One can find several interesting other explicit values of $V(q)$ using the values of $a_{M, 3}, a_{M, 9}, b_{M, 3}$ and $b_{M, 9}$ in [10], [11], [12] and [13].

Acknowledgment. The authors are thankful to the referee for their valuable suggestions which improves the quality of the paper. The first and the last authors are thankful to DST for their support under the research project SR/S4/MS:509/07.

\section{References}

[1] C. Adiga, T. Kim, M. S. Mahadeva Naika and H. S. Madhusudhan, On Ramanujan's cubic continued fraction and explicit evaluations of theta-function, Indian J. Pure and Appl. Math., 35(9)(2004), 1047-1062.

[2] C. Adiga, K. R. Vasuki, and M. S. Mahadeva Naika, Some new explicit evaluations of Ramanujan's cubic continued fraction, The New Zealand J. Math., 31(2002), 1-6.

[3] N. D. Baruah, Modular equations for Ramanujan's cubic continued fraction, J. Math. Anal. and Appl., 268(2002), 244-255.

[4] N. D. Baruah and Nipen Saikia, Some general theorems on the explicit evaluations of Ramanujan's cubic continued fraction, J. Compu. and Appl. Math., 160(2003), 37-51.

[5] B. C. Berndt, Ramanujan's Notebooks, Part III, Springer-Verlag, New York, 1991.

[6] B. C. Berndt, H. H. Chan and L. -C. Zhang, Ramanujan's remarkable product of the theta-function, Proc. Edinburgh Math. Soc., 40(1997), 583-612.

[7] H. H. Chan, On Ramanujan's cubic continued fraction, Acta Arith., 73(1995), 343355 .

[8] M. S. Mahadeva Naika, $P-Q$ eta-function identities and computation of RamanujanWebber class invariants, J. Indian Math. Soc., 70(1-4), (2003), 121-134.

[9] M. S. Mahadeva Naika, Some theorems on Ramanujan's cubic continued fraction and related identities, Tamsui Oxford J. Math. Sci., 24(3)(2008), 243-256. 
[10] M. S. Mahadeva Naika and B. N. Dharmendra, On some new general theorems for the explicit evaluations of Ramanujan's remarkable product of theta-function, The Ramanujan J., 15(3)(2008), 349-366.

[11] M. S. Mahadeva Naika, B. N. Dharmendra and K. Shivashankara, On some new explicit evaluations of Ramanujan's remarkable product of theta-function, South East Asian J. Math. and Math. Sci., 5(1)(2006), 107-119.

[12] M. S. Mahadeva Naika and M. C. Maheshkumar, Explicit evaluations of Ramanujan's remarkable product of theta-function, Adv. Stud. Contemp. Math., 13(2)(2006), 235254.

[13] M. S. Mahadeva Naika, M. C. Maheshkumar and K. Sushan Bairy, On some remarkable product of theta-function, Aust. J. Math. Anal. Appl., 5(1)(2008), Art. 13, $1-15$.

[14] S. Ramanujan, Notebooks (2 volumes), Tata Institute of Fundamental Research, Bombay, 1957.

[15] S. Ramanujan, The lost notebook and other unpublished papers, Narosa, New Delhi, 1988. 